\title{
High Pressure in Boron Nitride Nanotubes for Kirigami Nanoribbon Elaboration
}

Silvio D. Silva-Santos, ${ }^{\dagger}, \#$ Anthony Impellizzeri, ${ }^{\ddagger}$ Acrisio L. Aguiar, $"$ Catherine Journet, ${ }^{\S}$ Charline Dalverny, $\|$ Bérangère Toury, ${ }^{\S}$ José M. De Sousa, ${ }^{\perp}$ Chris $\mathrm{P}$. Ewels, ${ }^{\ddagger}$ and Alfonso San-Miguel ${ }^{*, \dagger}$

†Université de Lyon, F-69000 Lyon, France and Institut Lumière Matière, CNRS, UMR 5306, Université Lyon 1, F-69622 Villeurbanne, France

$\ddagger$ Université de Nantes, CNRS, Institut des Matériaux Jean Rouxel, F-44000 Nantes, France

ФDepartamento de Física, Universidade Federal do Piauí, Teresina, Piauí, 64049-550,

\section{Brazil}

$\S$ Université de Lyon, F-69000 Lyon, France and Laboratoire Multimatériaux et Interfaces, CNRS, UMR 5615, Université Lyon 1, F-69622 Villeurbanne, France

|| Université de Lyon 1, F-69000 Lyon, Centre Technologique des Microstructures, 5 rue Raphael Dubois, 69622 Villeurbanne Cedex, France

$\perp$ Instituto Federal do Piauí - IFPI, São Raimundo Nonato, Piauí, 64770-000, Brazil

\#Current address: Electrical Engineering Department, Laboratory of Nanoelectronics and Nanophotonics, Federal University of Pará, PO Box 8619, Agencia UFPA, CEP 66075-900 Belém, Pará, Brazil

E-mail: alfonso.san-miguel@univ-lyon1.fr 


\section{Supporting Information}

\section{Laser Heating Effect on BNNTs Samples}

Figure $\$ 1$ shows the effect of laser heating on the BNMWNT sample. A air-cooled argonion laser operating at the $514.5 \mathrm{~nm}$ wavelength was used. No significant changes related to the displacement of the tangential BNMWNT mode is observed. Only parameters such as spectral profiles, background and signal-to-noise ratio showed variations between spectra. So, the laser power of $20 \mathrm{~mW}$ was selected to run our experiments.

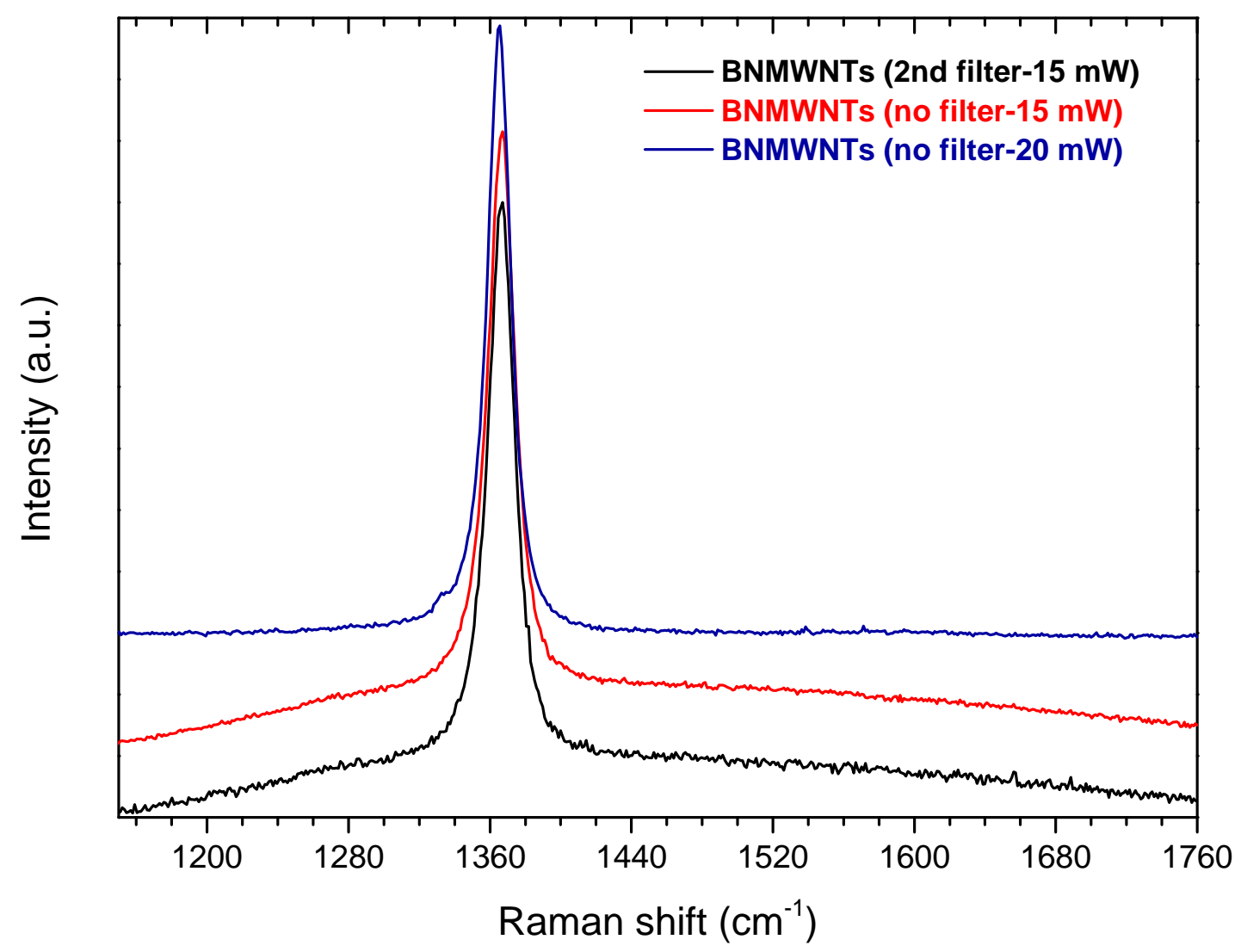

Figure S 1: Tangential modes comparison of BNMWNTs using an intermediate filter and no filter at the excitation energy of $2.41 \mathrm{eV}$. 


\section{Tomography Videos}

Electron Tomography videos of the pristine and after $36 \mathrm{GPa}$ BNNTs spinning around their tube axes (in the vertical position) can be seen at (1) and (2), respectively:

1. Pristine MWBNNT:https://drive.google.com/file/d/1360mh9cr2ipIokiCgk1C0hwbydjARSdf/ view?usp=sharing

2. MWBNNT after pressure cycle up to 36 GPa: https://drive.google.com/file/d/ 16w1o8cBGRTczh_vCn1uEhU5WFCSAK4f1/view?usp=sharing

The cross-sections videos for the tubes aforementioned were taken in relation to each vertical position and the snapshots series have been evolved along the tube from down to up. These movies may be downloaded from:

- BNNT-pristine cross-section: https://drive.google.com/file/d/1ghwxxY14wMHQS7Lw7rxrgMu2DdDpp3UL/view?usp= sharing

- BNNT after 36 GPa cross-section: https://drive.google.com/file/d/1Lt3EWyD8HhL7h06CZ3SwlhptMTAOg75h/view?usp= sharing

Next, we present a dark spot pattern tomography video of a pristine BNNT tube obtained by a tilt series of this specimen from $-59^{\circ}$ to $65^{\circ}$ taken at $200 \mathrm{keV}$ acceleration. The dark spots do not present a regular pitch periodically alternated on the tube opposite sides in

agreement with the previous findings of Golberg et al. 1 The movie showing the dark spots evolution of the pristine BNNT studied in our work can be accessed below:

https://drive.google.com/file/d/1DPy-1P0ry3PM1fH0jpJqDh5jbQutIjAU/view?usp= sharing 


\section{Raman Spectroscopy Results}
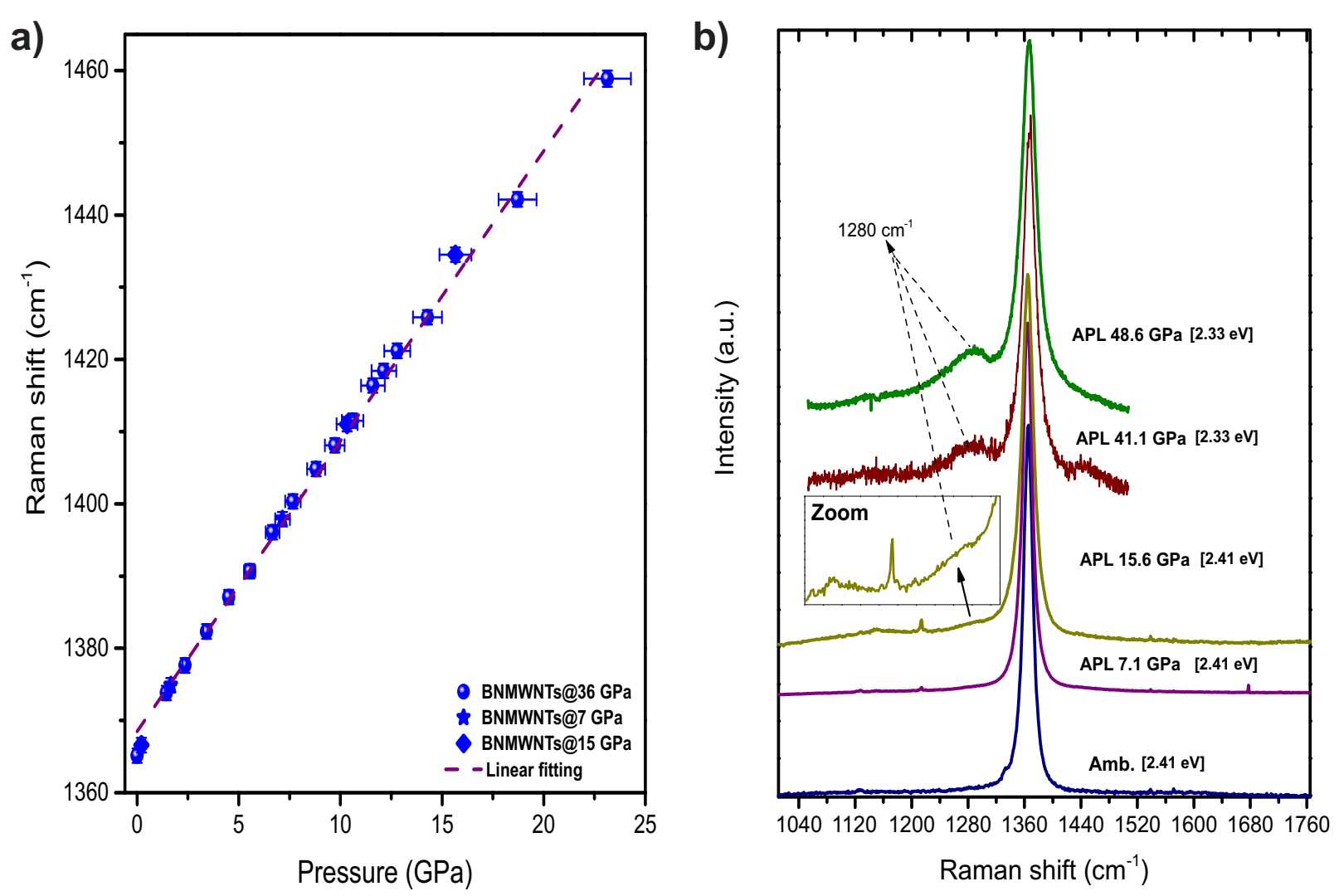

Figure S 2: High pressure evolution of the the $E_{2 g}$ tangential mode in MWBNNTs. a) Frequency of the peak for 3 different experiments with 7, 15 and 36 GPa maximum pressures respectively. The dashed line corresponds to the linear fit of all the data. b) Comparison of the Raman spectra of the MWBNNTs TM signal at ambient conditions and after a pressure load cycle (APL) with maximum pressures of 7.1, 15.6, 41.1 and 48.6 GPa. Spectra are vertically shifted for clarity. The excitation wavelength is indicated between brackets. 


\section{Molecular Dynamics Simulation of BNNTs Under High Pressure}

For thermalization at room-temperature, we used 3-, 4-, 5-walled zigzag $(\mathrm{n}, 0) \mathrm{BN}$ nanotubes in diameter range of $0.78 \mathrm{~nm}-12.48 \mathrm{~nm}$ for inner tubes. Those multi-layered $\mathrm{BN}$ nanotubes were constructed using a $(n, 0) @(n+8,0)$ relation which was found to give a optimal energetic coupling. For thermalization of isolated few-layer BNNTs, periodic conditions were used only in tube axis (z-axis) direction with four unit cells $(\sim 1.704 \mathrm{~nm})$. The dynamic thermalization coupling at $300 \mathrm{~K}$ of all simulated BNNTs were performed by Nose-Hoover thermostat (implemented in LAMMPS code) $)^{2}$ during $2.10^{5} \mathrm{fs}$, where timestep of all dynamics simulations is $0.05 \mathrm{fs}$. In order to minimize the periodic effect of use short tube supercells, we also performed the thermalization of a long 3-walled BNNT $(20,0) @(28,0) @(36,0)$, with 20 and 40 unit cells $(8.52 \mathrm{~nm}$ and $17.04 \mathrm{~nm}$, respectively). For the high-compression modelling, the $(20,0) @(28,0) @(36,0) 3$-walled BN nanotube was arranged in a triangular symmetric bundle. In this case we use uniaxial compression in order to see the effect of high-pressure conditions on inner, intermediate and outer tubes of this 3-walled BNNT. The applied compression on MWBNNTs bundles was simulated by gradual decrease of an orthogonal simulation box (x-axis) at a rate of $10^{-6} / f s$, which means that the box dimension changes linearly in time, from its initial to final values. For each MD step, we collected the stress-tensor component

$\left(\sigma_{x x}\right)$ and we associated with the applied uniaxial pressure. The maximum uniaxial pressure reached in our model was $107 \mathrm{GPa}$. Periodic conditions were also used in high-pressure compression model for all directions. 

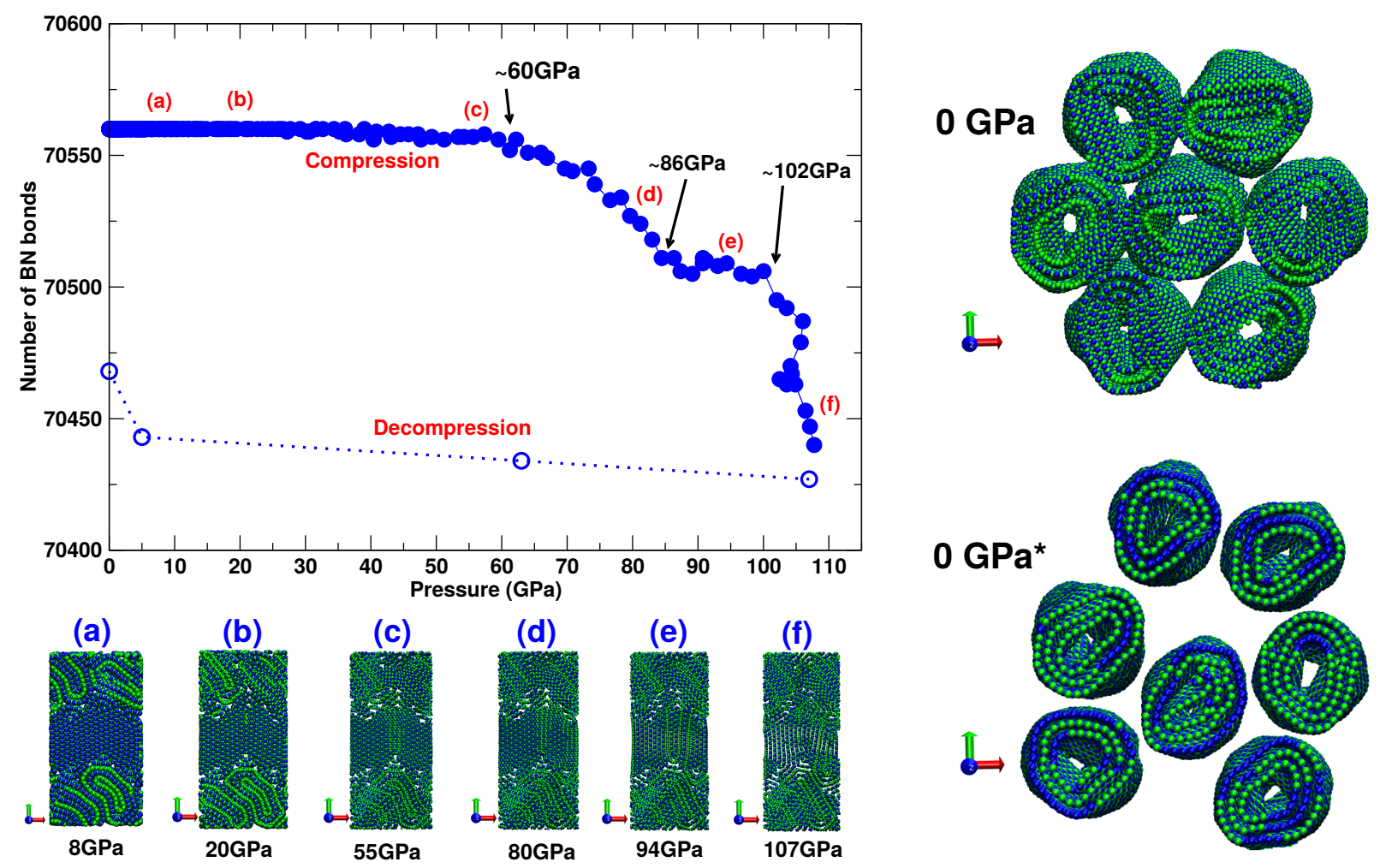

Figure S 3: Structural evolution of the $(20,0) @(28,0) @(36,0)$ BNNT under uniaxial compression cycle. Top panel: The number of BN bonds as a function of applied pressure during the compression and decompression process. The maximum pressure reached in this cycle was 107GPa. Arrows indicates pressure values where we observed different trends. Down panels: (a)-(f) snapshots of BWNT bundle as the pressure is increased. Right panels: The $(20,0) @(28,0) @(36,0)$ bundle before $(0 \mathrm{GPa})$ and after $\left(0 \mathrm{GPa}^{*}\right)$ the pressure cycle. 


\section{Interlayer Interactions in $\mathrm{hBN}$ and Graphite}

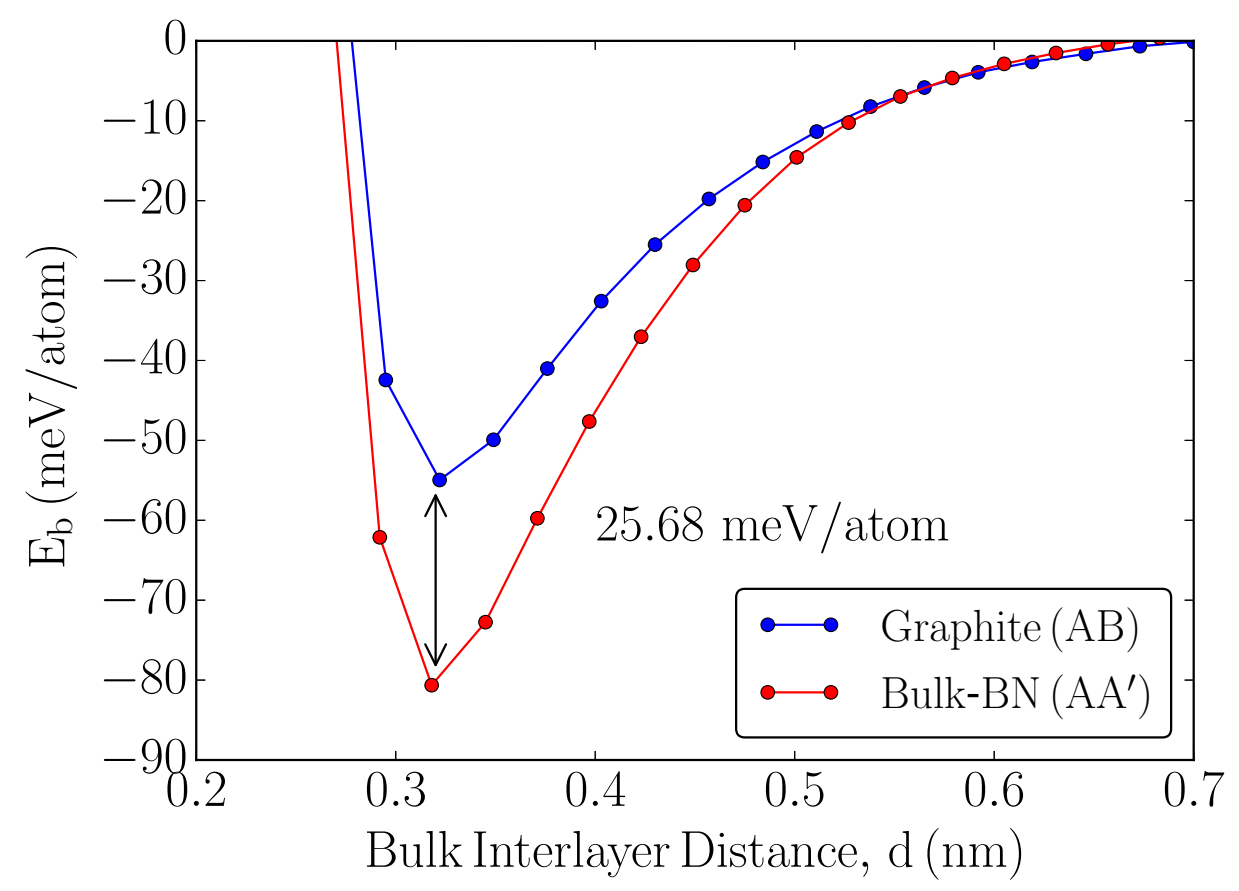

Figure S 4: Density functional theory (DFT) calculated interlayer binding enthalpy for Graphite (blue line) and h-BN (red line) using GGA-PBE+D2 theory level as a function of interlayer distance, in most stable stacking configurations.

\section{Structure and Energetics of Collapsed SWBNNTs}

We have considered a series of different diameter $(\mathrm{D})$ armchair $(n, n)$ and zig-zag $(n, 0)$ singlewalled BNNTs and calculated the total energy difference between collapsed ( $\mathrm{E}_{\text {tot }}^{\text {collapsed }}$ ) and circular $\left(\mathrm{E}_{\text {tot }}^{\text {circular }}\right)$ shape in order to determine accurately at what threshold diameter fully flattened BN tubes become the most stable configuration.

For these free-standing nanotubes, we found two distinct critical diameters: $\mathrm{D}_{\text {meta }}$ and $\mathrm{D}_{\text {cross. }}$. All nanotubes with diameters $\mathrm{D}<\mathrm{D}_{\text {meta }}$ are characterized by circular shape. For tubes with $\mathrm{D}_{\text {meta }}<\mathrm{D}<\mathrm{D}_{\text {cross }}$ can assume a collapsed peanut- or dumbbell-like (depending on the width of the central flat region), but they are energetically less stable in comparison to cylindrical ones. 


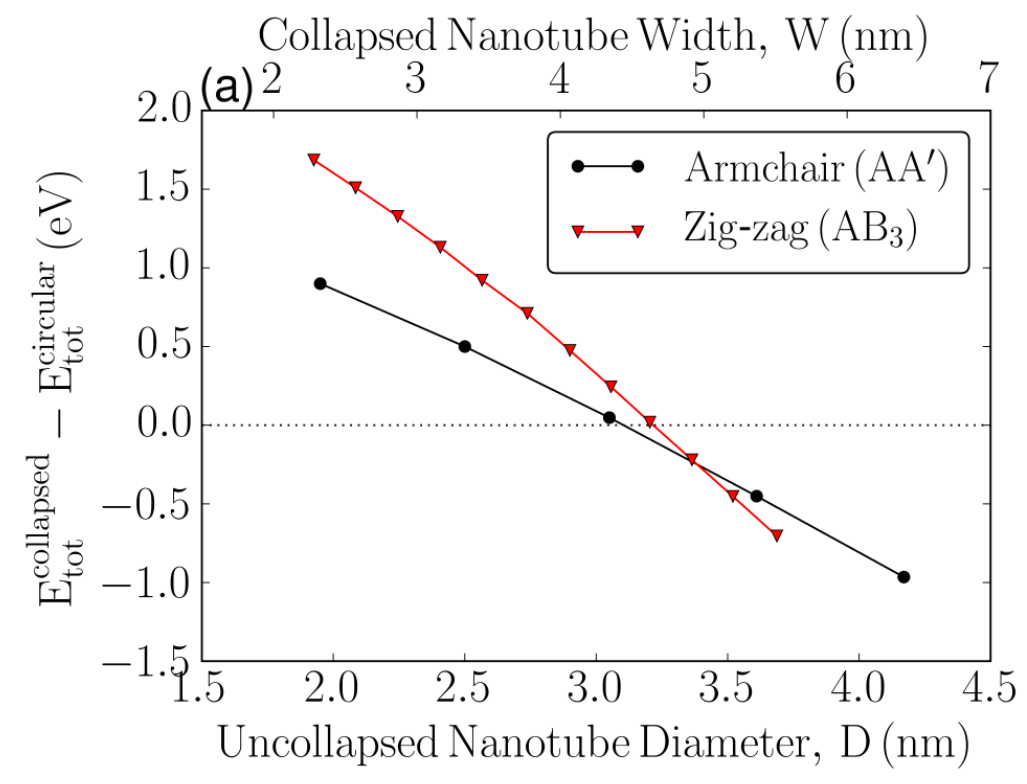

(b) Armchair

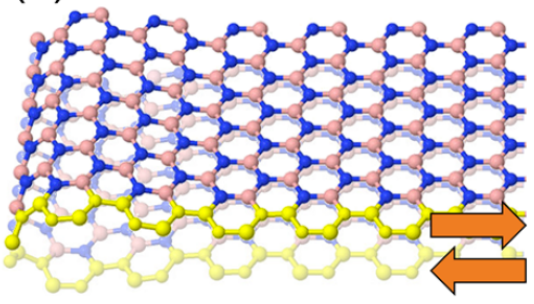

(c) Zig-zag

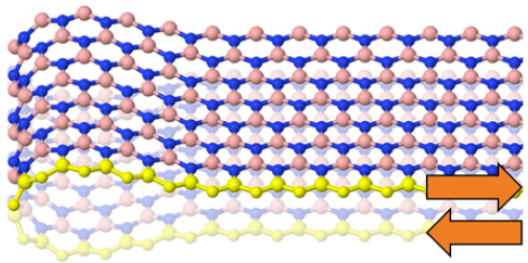

Figure S 5: (a) GGA-D2 calculated total energy difference between free-standing collapsed $\left(\mathrm{E}_{\text {tot }}^{\text {collapsed }}\right)$ and circular $\left(\mathrm{E}_{\text {tot }}^{\text {circular }}\right)$ armchair (black line) and zig-zag (red line) SWCNTs versus tube diameter $\mathrm{D}$ (lower $x$-axis) and equivalent width (upper $x$-axis). The intersection point at $\Delta E_{\text {tot }}=0.0 \mathrm{eV}$ indicates the threshold diameter above which collapsed boron-nitride nanotubes become energetically favourable in comparison to circular ones. Schematic illustration of collapsed tubes with (b) armchair and (c) zigzag edges, whose most energetically favored central flattened zones are arranged in $\mathrm{AA}^{\prime}$ - and $\mathrm{AB}_{3}$-stackings, respectively. Yellow balls indicated nanotube orientation, while red arrows show interlayer shearing mode.

The collapsed nanotubes with $\mathrm{D}>\mathrm{D}_{\text {cross }}$ are more favourable with respect to circular ones. Figure $\$ 5(a)$ shows that armchair and zig-zag collapsed nanotubes are stabilised at diameter values $\mathrm{D}_{\text {cross }}=3.05 \mathrm{~nm}$ and $3.20 \mathrm{~nm}$, respectively. This result demonstrates that radial flattening deformation is largely independent by nanotube chirality. In our calculations, the lowest energy interlayer stacking was considered. The central flat zone of armchair nanotubes is characterized by $\mathrm{AA}^{\prime}$-stacking, while the overlapping flat layers of zig-zag family 

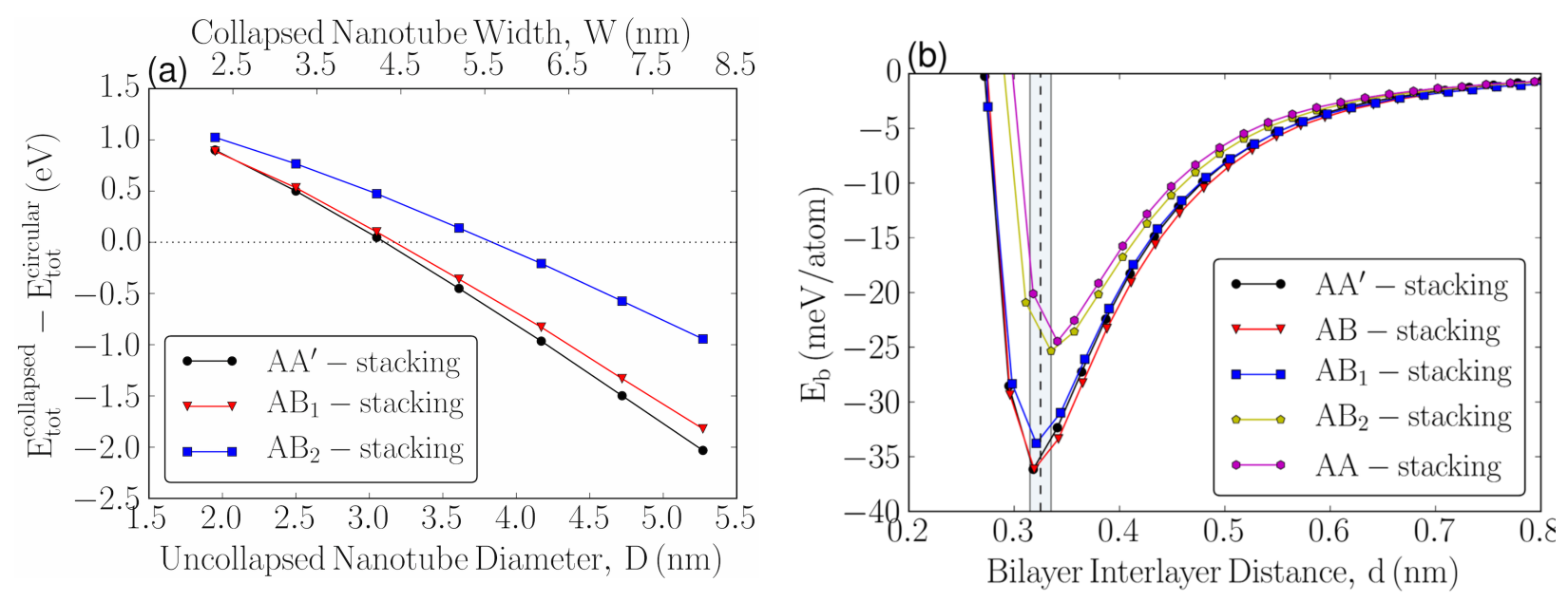

Figure S 6: (a) GGA-D2 calculated total energy difference between free-standing collapsed ( $\mathrm{E}_{\text {tot }}^{\text {collapsed }}$ ) and circular $\left(\mathrm{E}_{\text {tot }}^{\text {circular }}\right)$ armchair $(n, n)$ SWCNTs versus tube diameter $\mathrm{D}$ (lower $x$-axis) and equivalent width (upper $x$-axis). The intersection point at $\Delta E_{\text {tot }}=0.0 \mathrm{eV}$ indicates the threshold diameter above which collapsed boron-nitride nanotubes become energetically favourable in comparison to circular ones. Calculations are done by different stacking orientations: $\mathrm{AA}^{\prime}$ (black), $\mathrm{AB}_{1}$ (red), and $\mathrm{AB}_{2}$ (blue). (b) GGA-D2 calculated binding energies for infinite bilayer h-BN. Vertical dashed line and magenta section represent its average interlayer distance and corresponding uncertainty $(0.325 \pm 0.01 \mathrm{~nm})$ obtained in prior experimental studies.

are arranged in $\mathrm{AB}_{3}$-stacking, as depicted in Figure $\mathrm{S} 5(\mathrm{~b}),(\mathrm{c})$, respectively.

The influence of lattice registry was investigated in armchair collapsed nanotubes, considering other stacking combinations, labelled $\mathrm{AB}_{1}$ and $\mathrm{AB}_{2}$ (see Figure $\mathrm{S} 6(\mathrm{a})$ ). These two stacking modes were obtained by increasing the translational shearing along the [10̄0] direction. Varying the stacking fault of collapsed armchair nanotubes, we can see that the collapsed threshold diameter does not change between $\mathrm{AA}^{\prime}$ - and $\mathrm{AB}_{1}$-stacked, while it increases to $3.89 \mathrm{~nm}$ for $\mathrm{AB}_{2}$-stacked. Calculations of remaining stacking faults ( $\mathrm{AB}$ and $\mathrm{AA}$ ) are not reported, because it is not possible to get the B-N covalent interlayer bonding at the edges. All of these threshold values obtained by changing either chirality or stacking tube are somewhat smaller than value obtained for carbon counterpart in most reliable and recent experimental and theoretical works, i.e. $\sim 5.1 \mathrm{~nm} . \frac{314}{14}$ This result confirms the high ionic nature of boron nitride and is greatly supported by smaller binding energies predicted for infinite flat bilayer h-BN, as shown in Figure $\mathrm{S} 6(\mathrm{~b})$. 
Enlarged views of the structures of Fig. 6 in the main paper

$$
\text { 3-wall }
$$

\section{$(10,0) @(18,0) @(26,0)$}

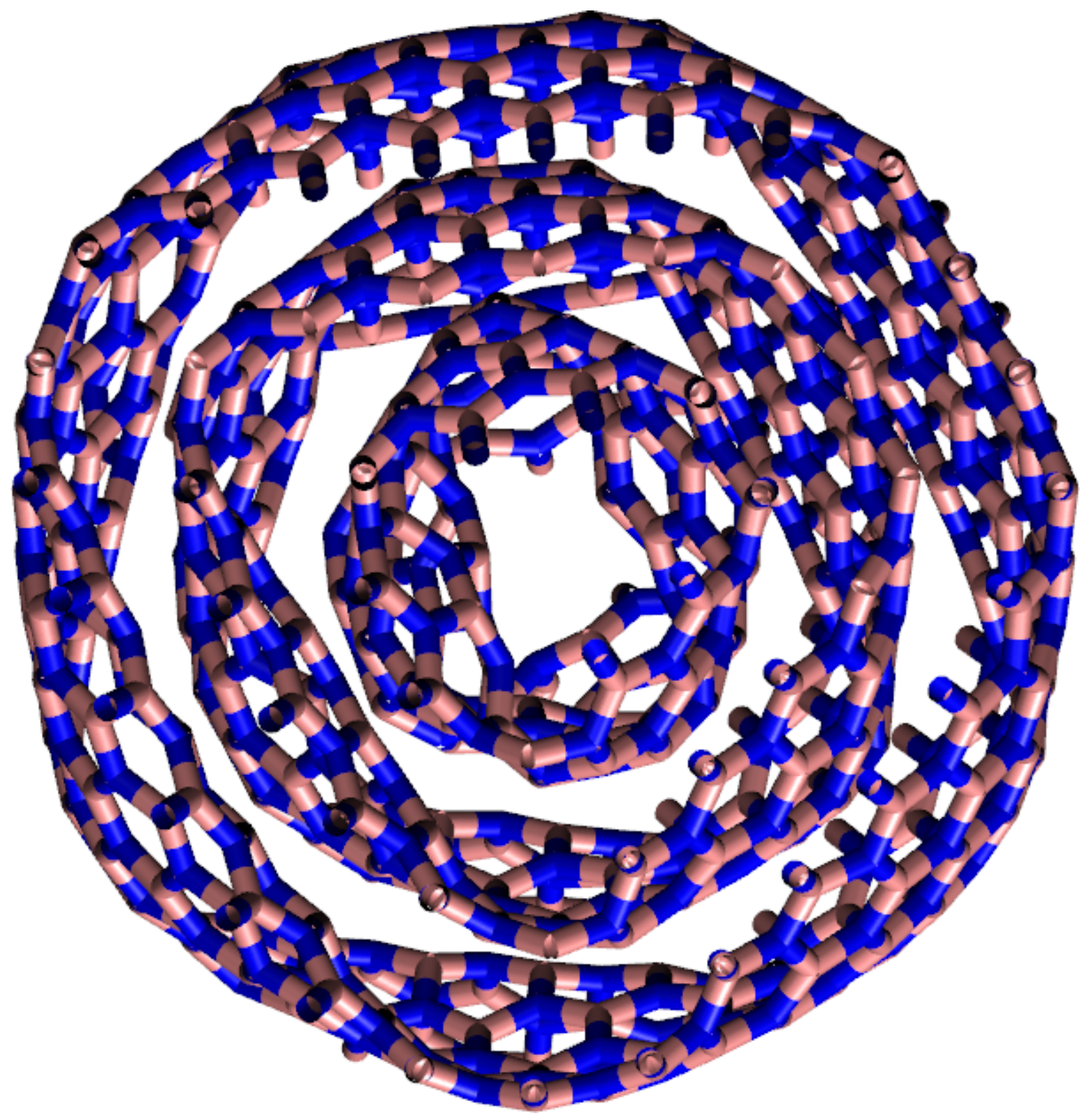




\section{3-wall}

\section{$(20,0) @(28,0) @(36,0)$}

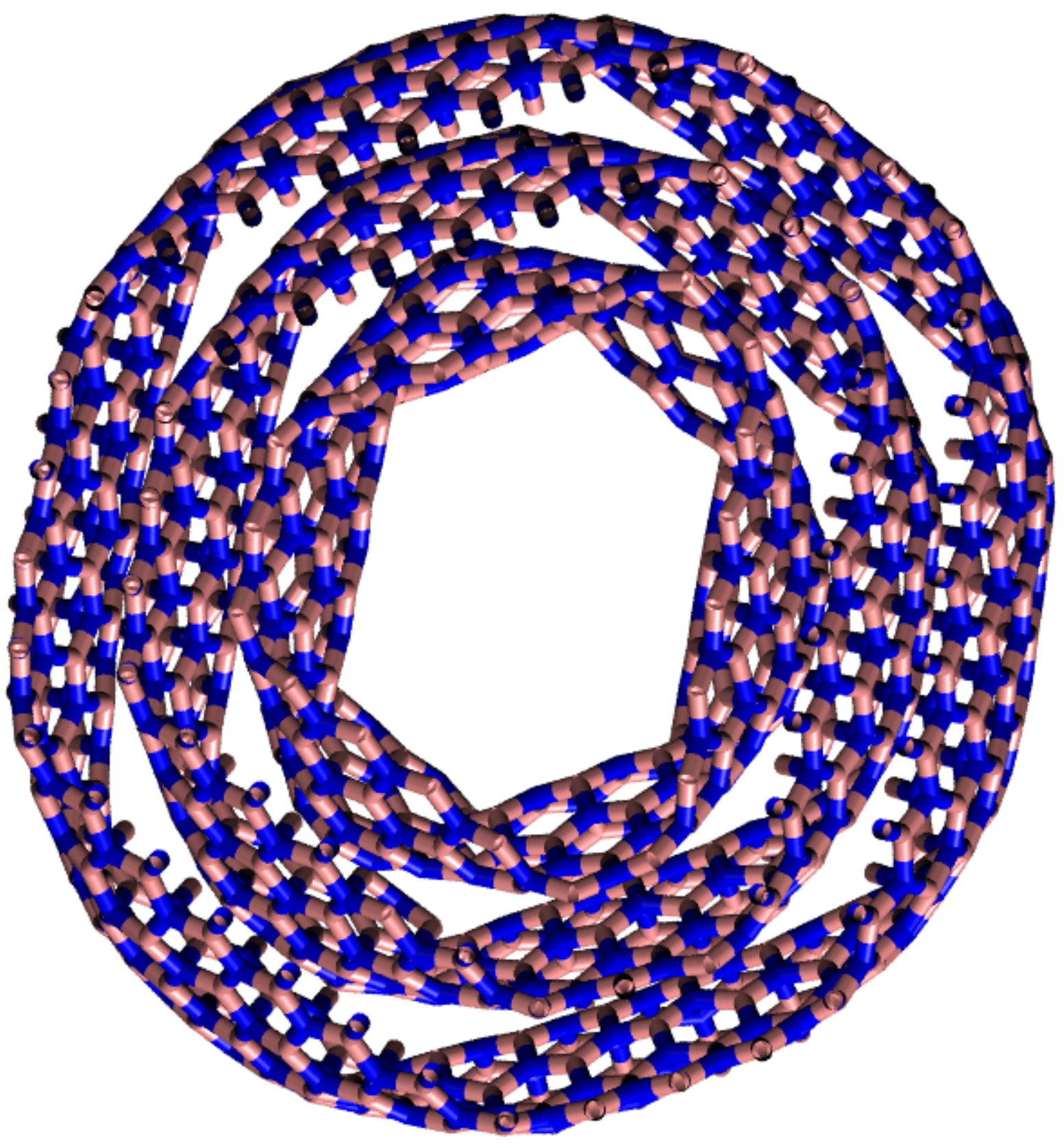




\section{3-wall}

\section{$(40,0) @(48,0) @(56,0)$}

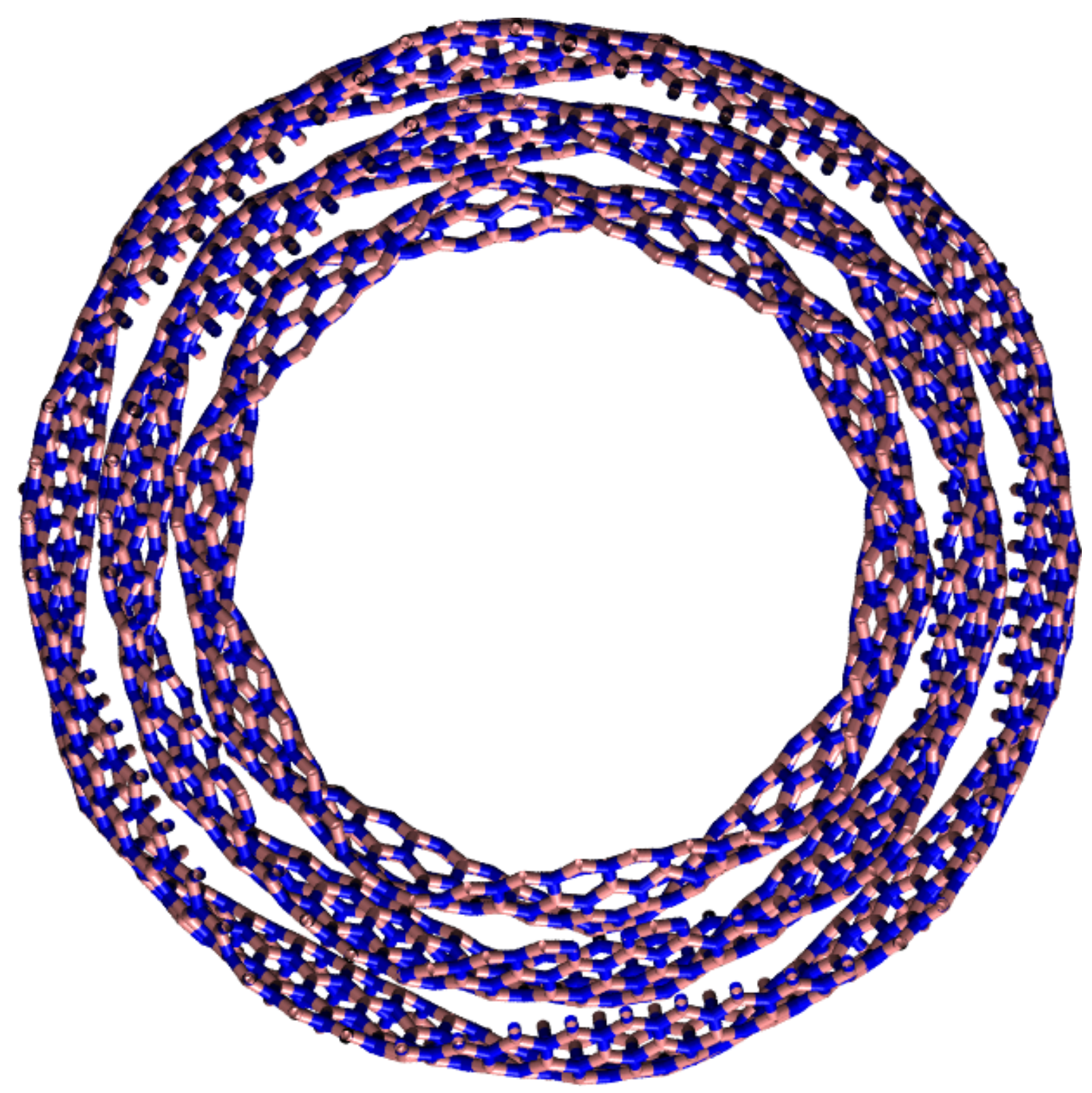




$$
\text { 3-wall }
$$

\section{$(80,0) @(88,0) @(96,0)$}

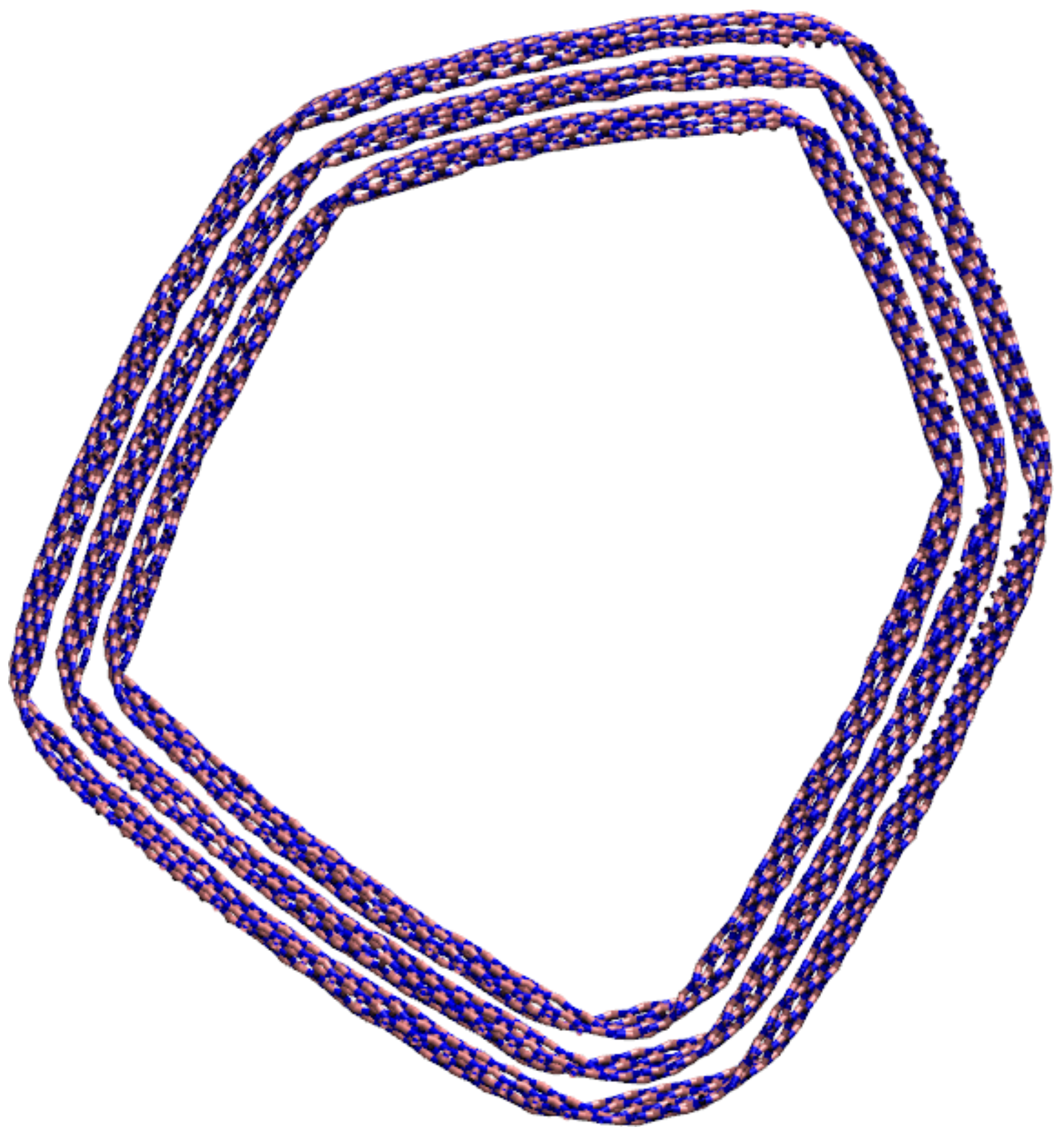




\section{3-wall}

\section{$(120,0) @(128,0) @(136,0)$}

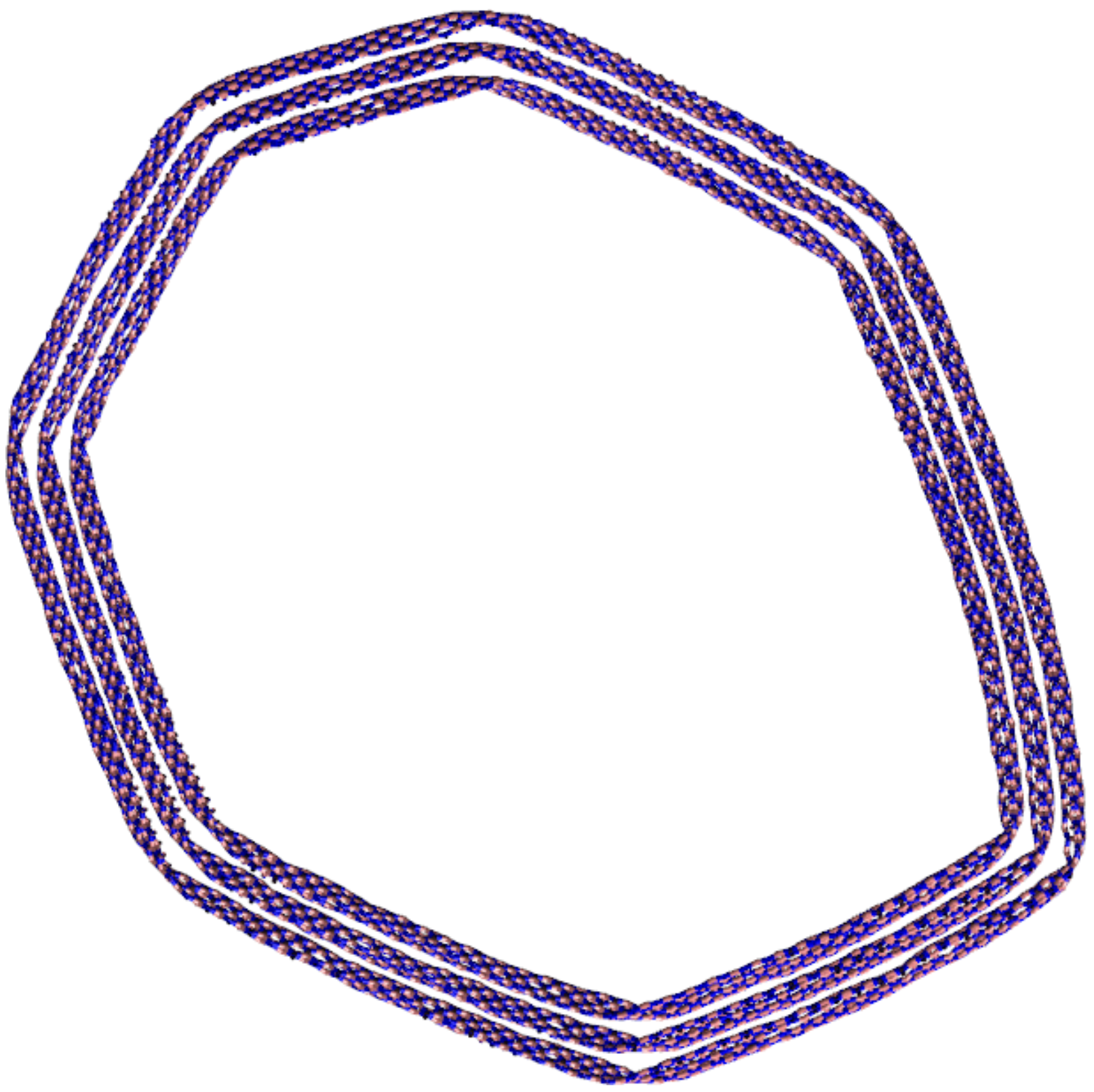




\section{3-wall}

\section{$(160,0) @(168,0) @(176,0)$}

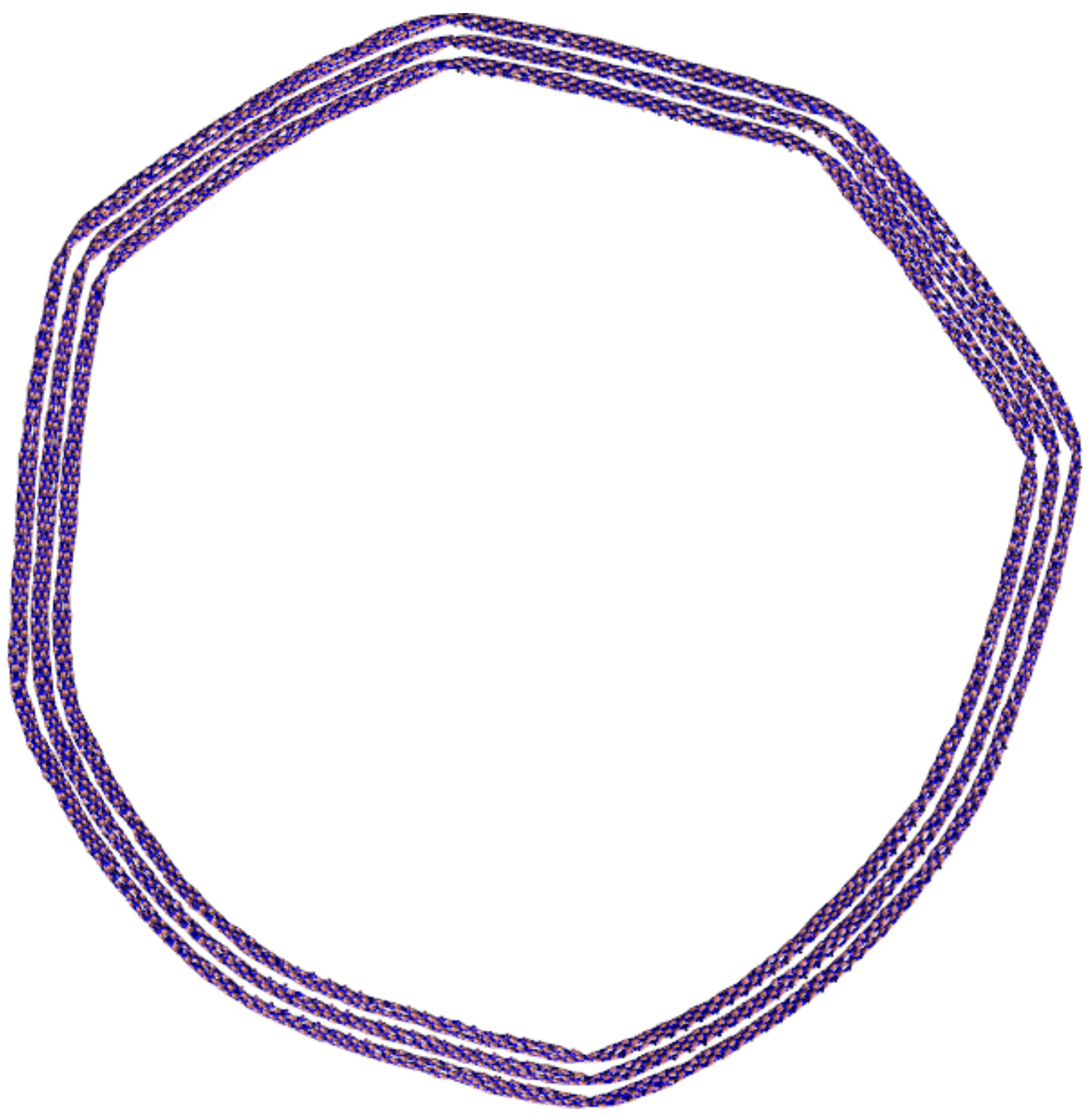




\section{4-wall}

\section{$(10,0) @(18,0) @(26,0) @(34,0)$}

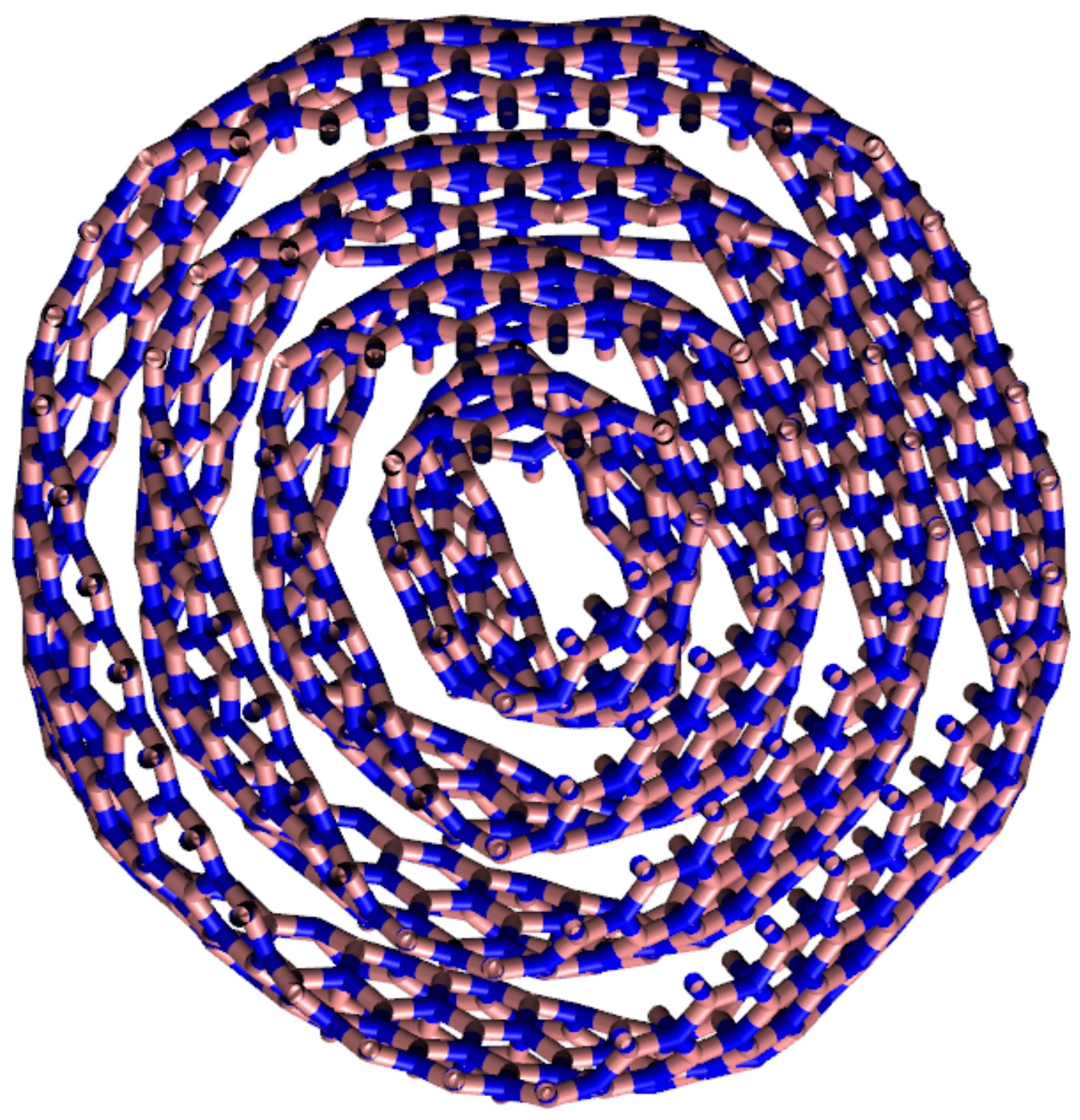




\section{4-wall}

\section{$(20,0) @(28,0) @(36,0) @(44,0)$}






\section{4-wall}

\section{$(40,0) @(48,0) @(56,0) @(64,0)$}

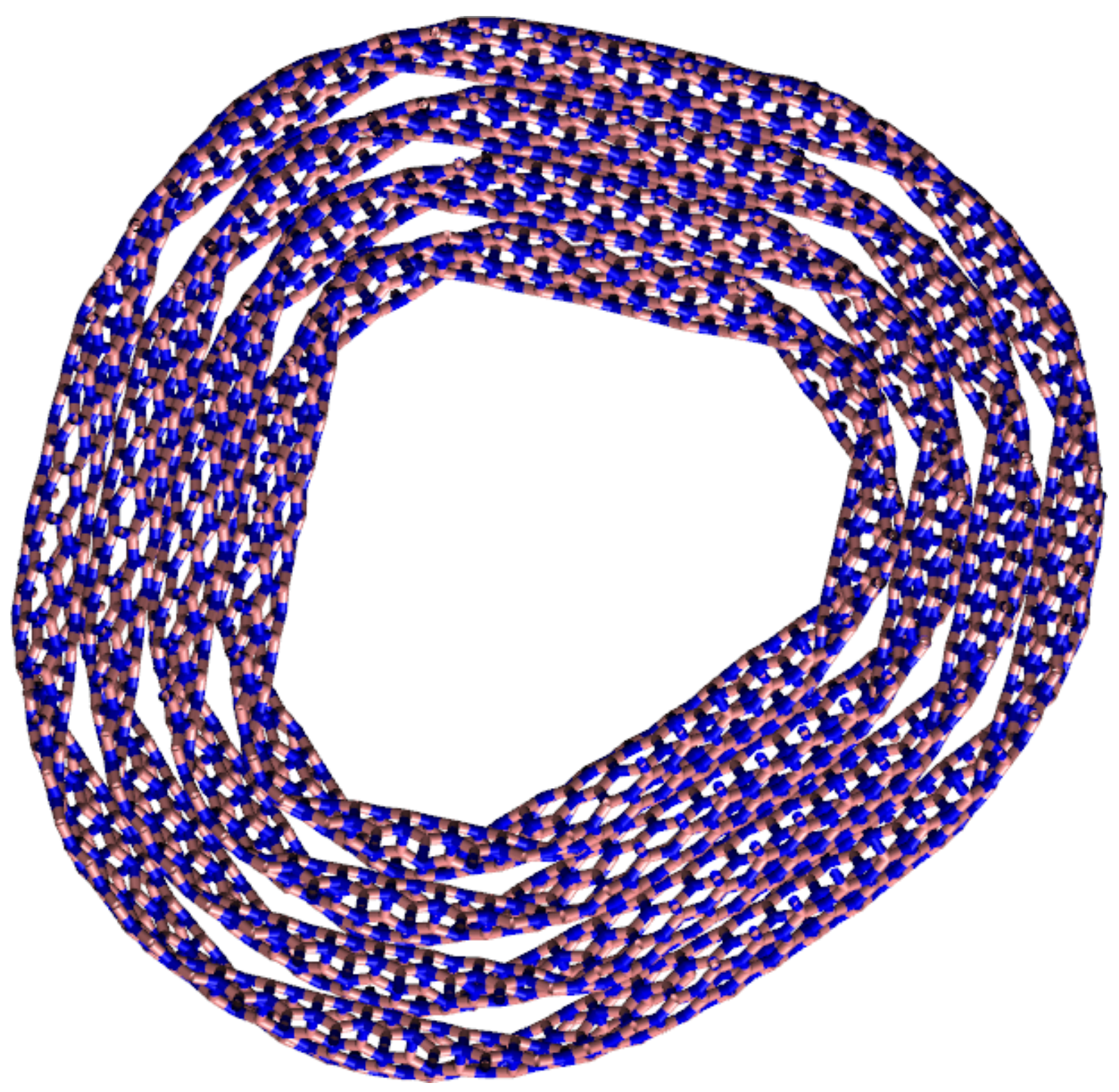




\section{4-wall}

\section{$(80,0) @(88,0) @(96,0) @(104,0)$}

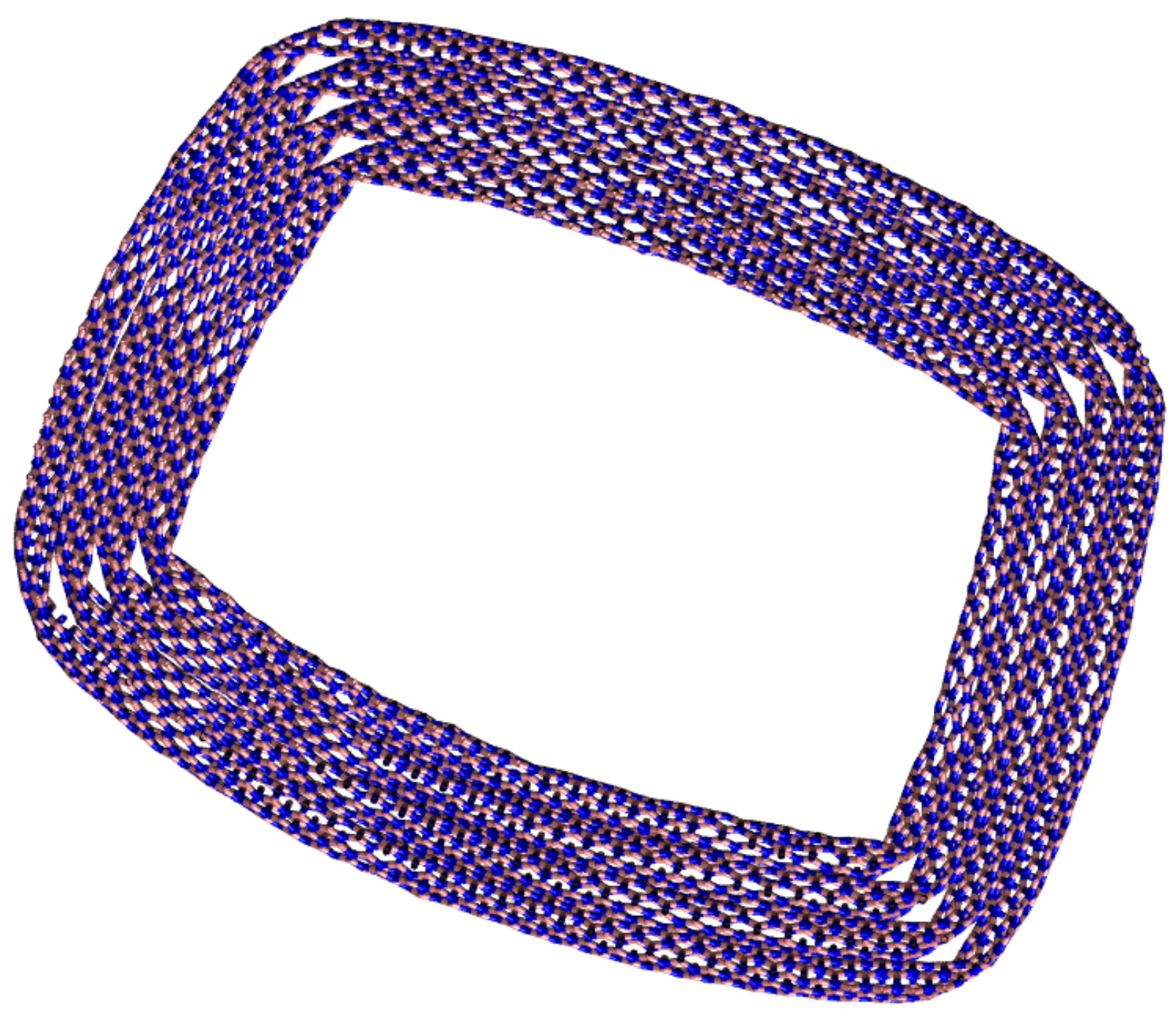




\section{4-wall}

\section{$(120,0) @(128,0) @(136,0) @(144,0)$}

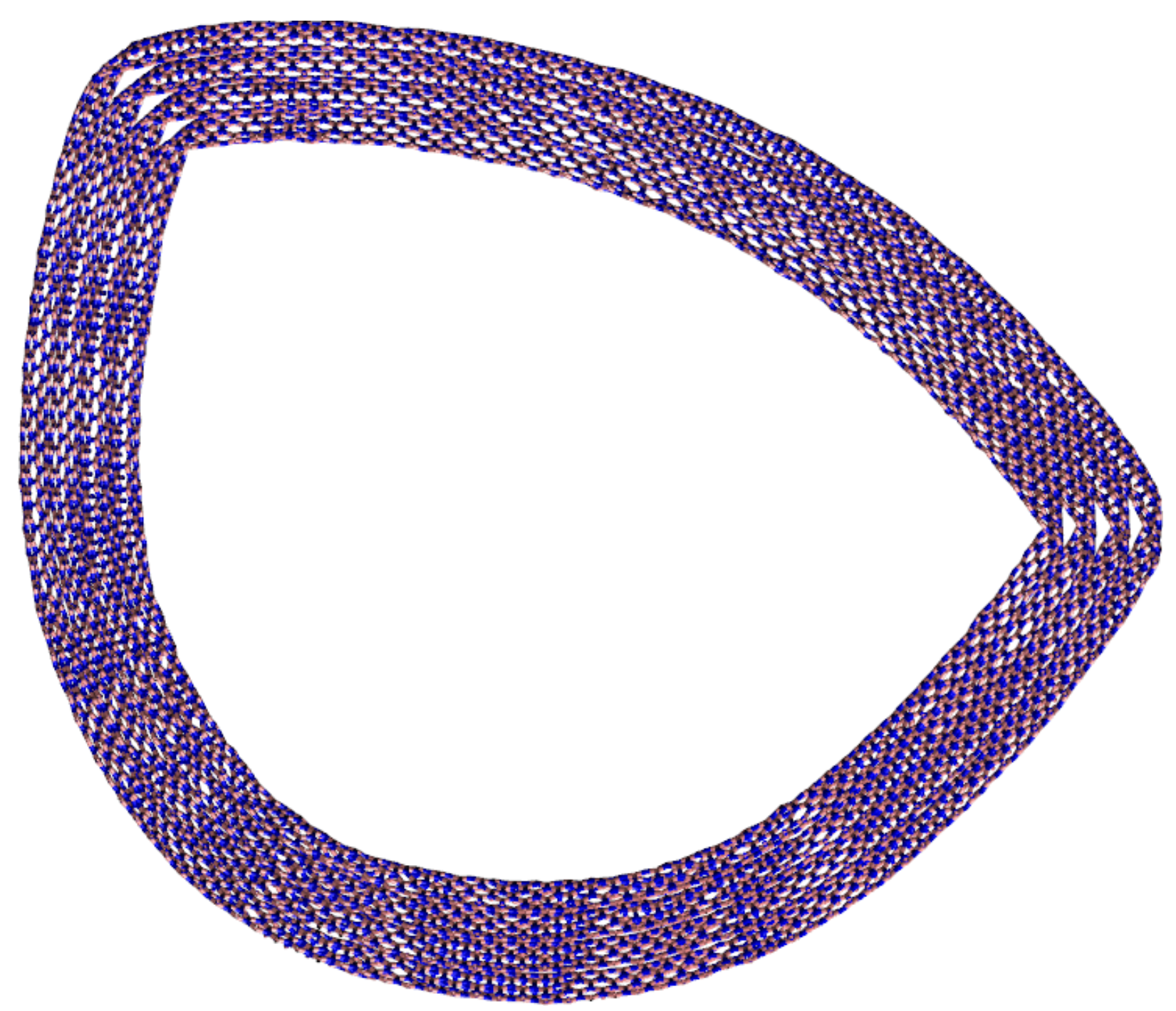




\section{4-wall}

\section{$(160,0) @(168,0) @(176,0) @(184,0)$}

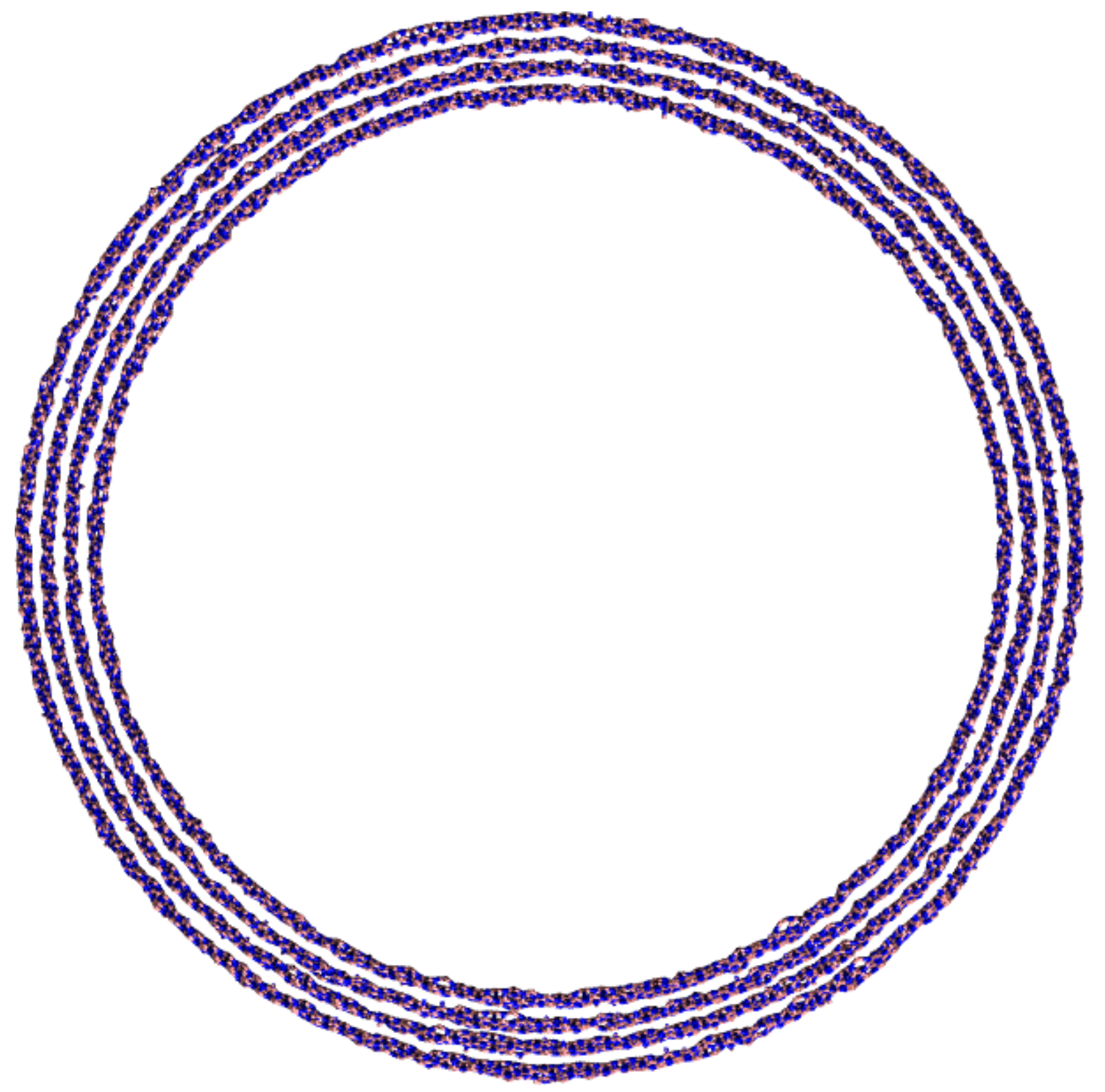




\section{5-wall}

\section{$(10,0) @(18,0) @(26,0) @(34,0) @(42,0)$}

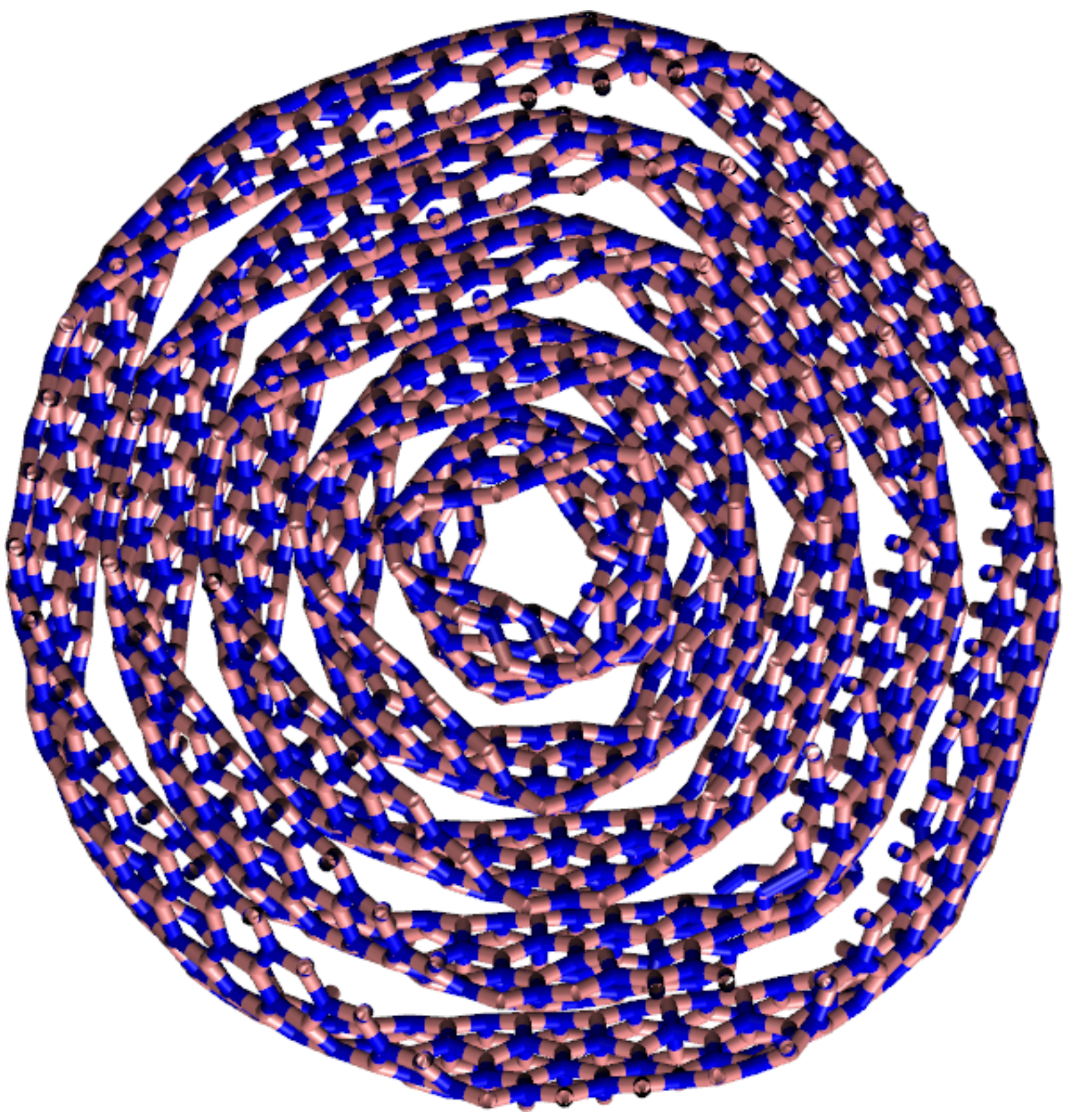




\section{5-wall}

\section{$(20,0) @(28,0) @(36,0) @(44,0) @(52,0)$}

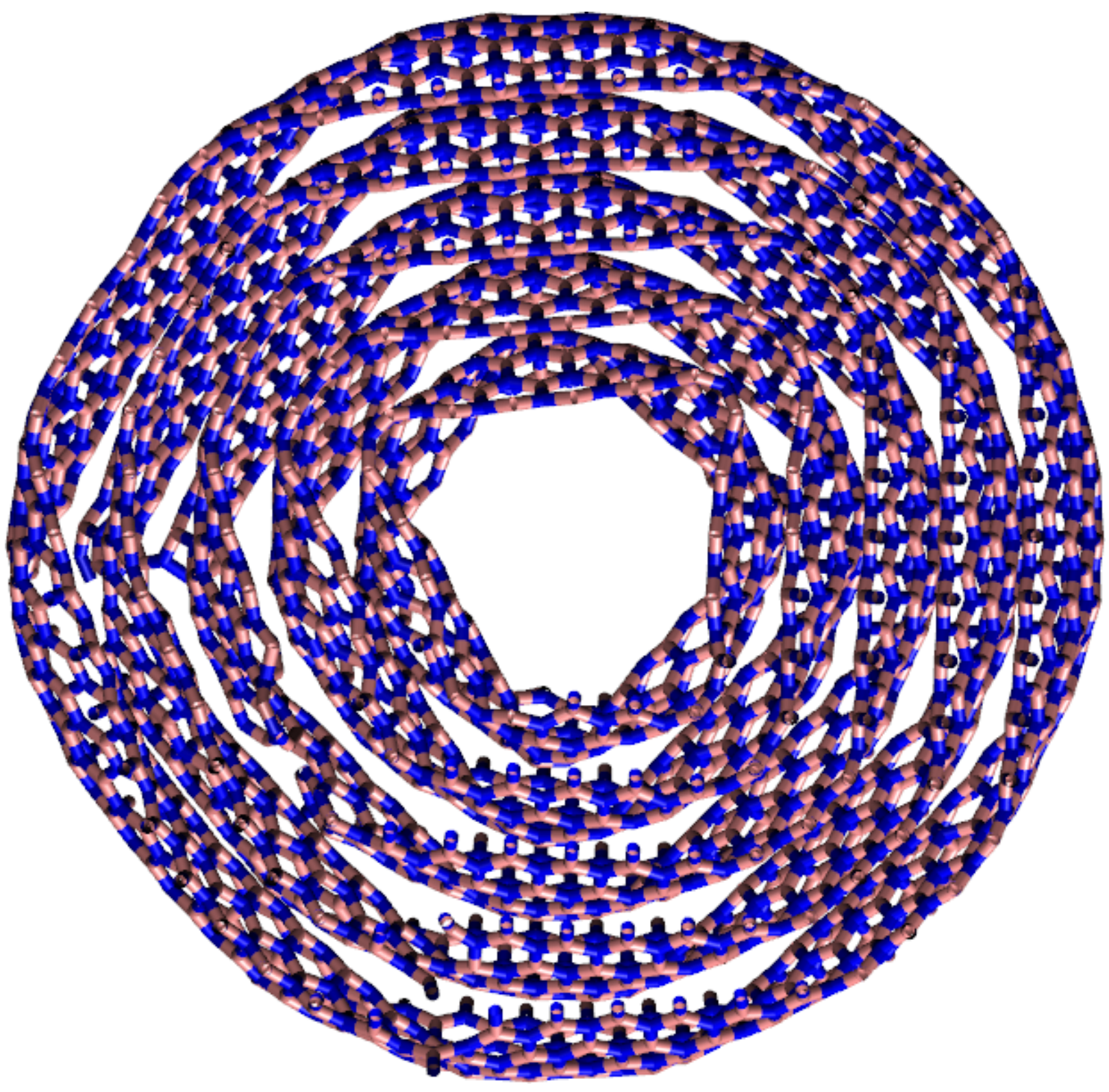




\section{5-wall}

\section{$(40,0) @(48,0) @(56,0) @(64,0) @(72,0)$}

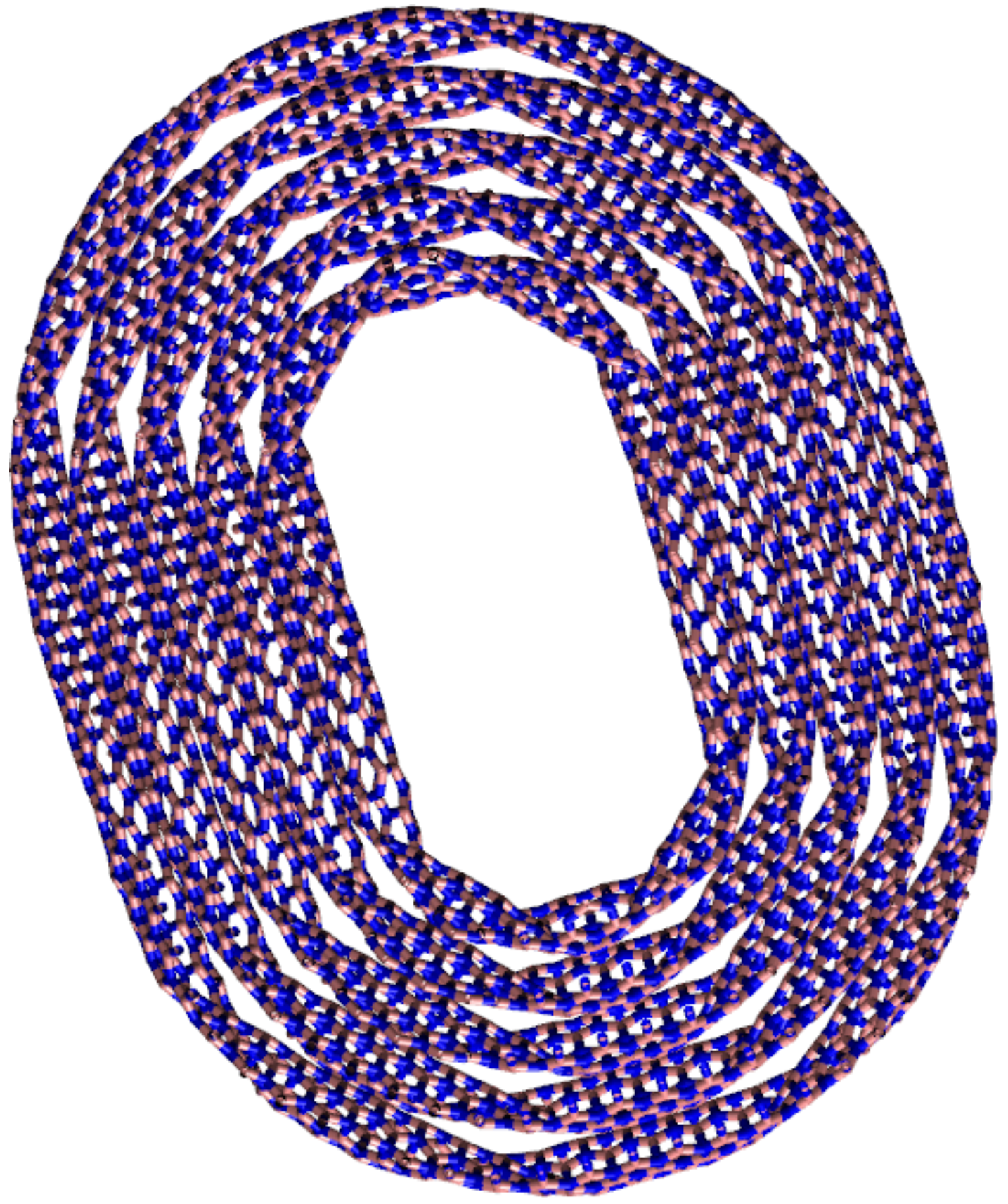




\section{5-wall}

\section{$(80,0) @(88,0) @(96,0) @(104,0) @(112,0)$}

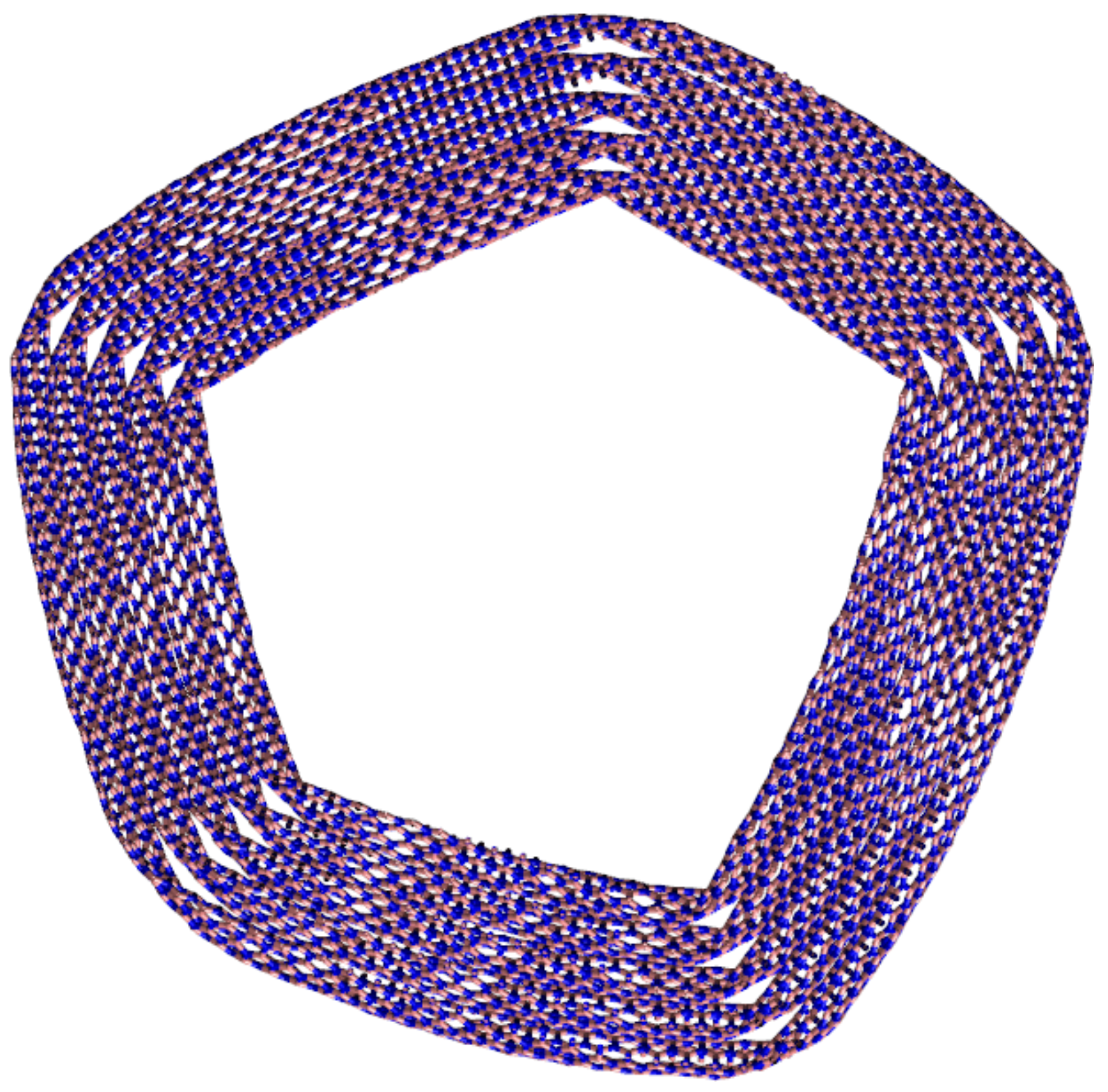




\section{5-wall}

\section{$(120,0) @(128,0) @(136,0) @(144,0) @(152,0)$}

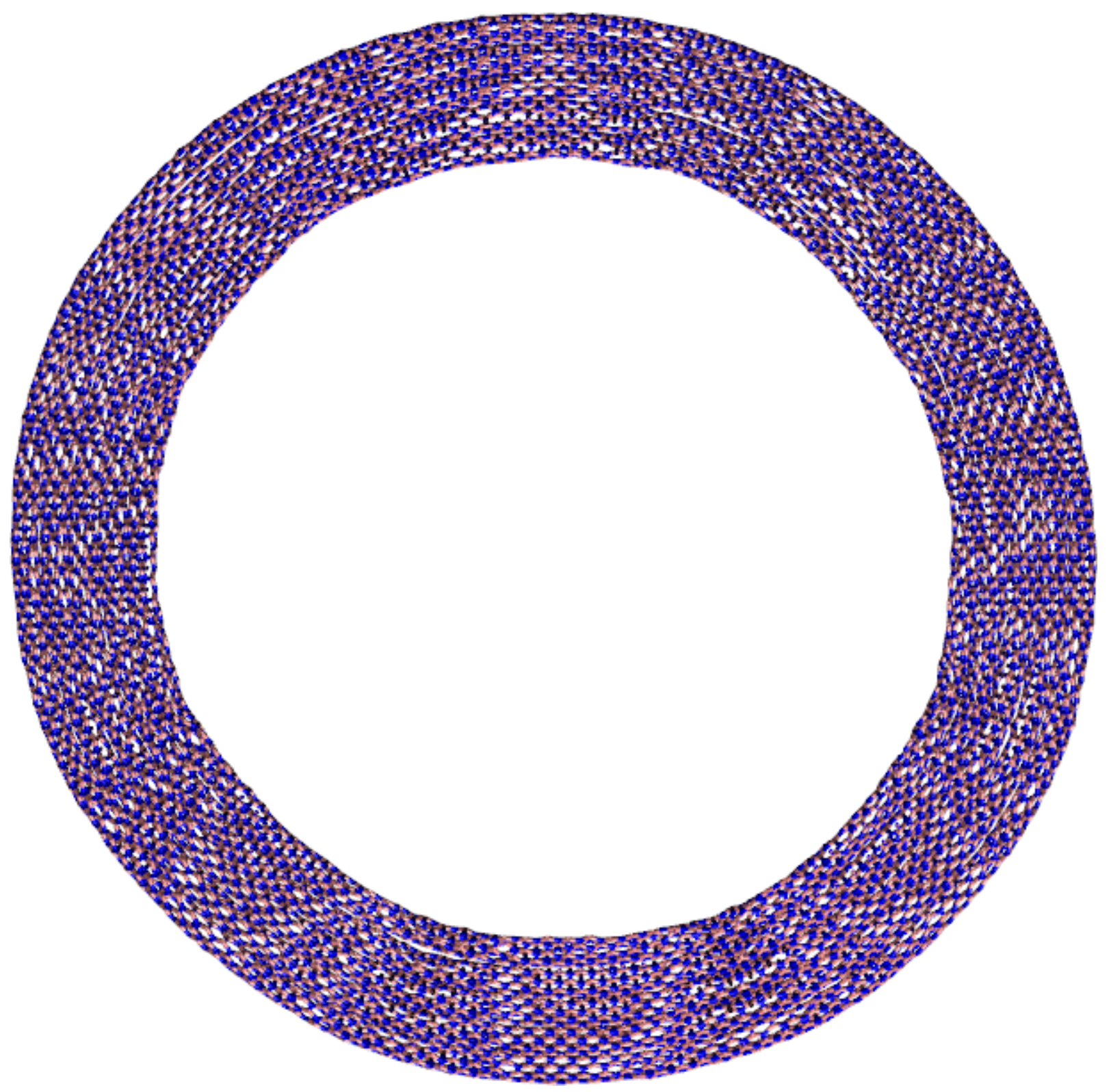




\section{5-wall}

\section{$(160,0) @(168,0) @(176,0) @(184,0) @(192,0)$}

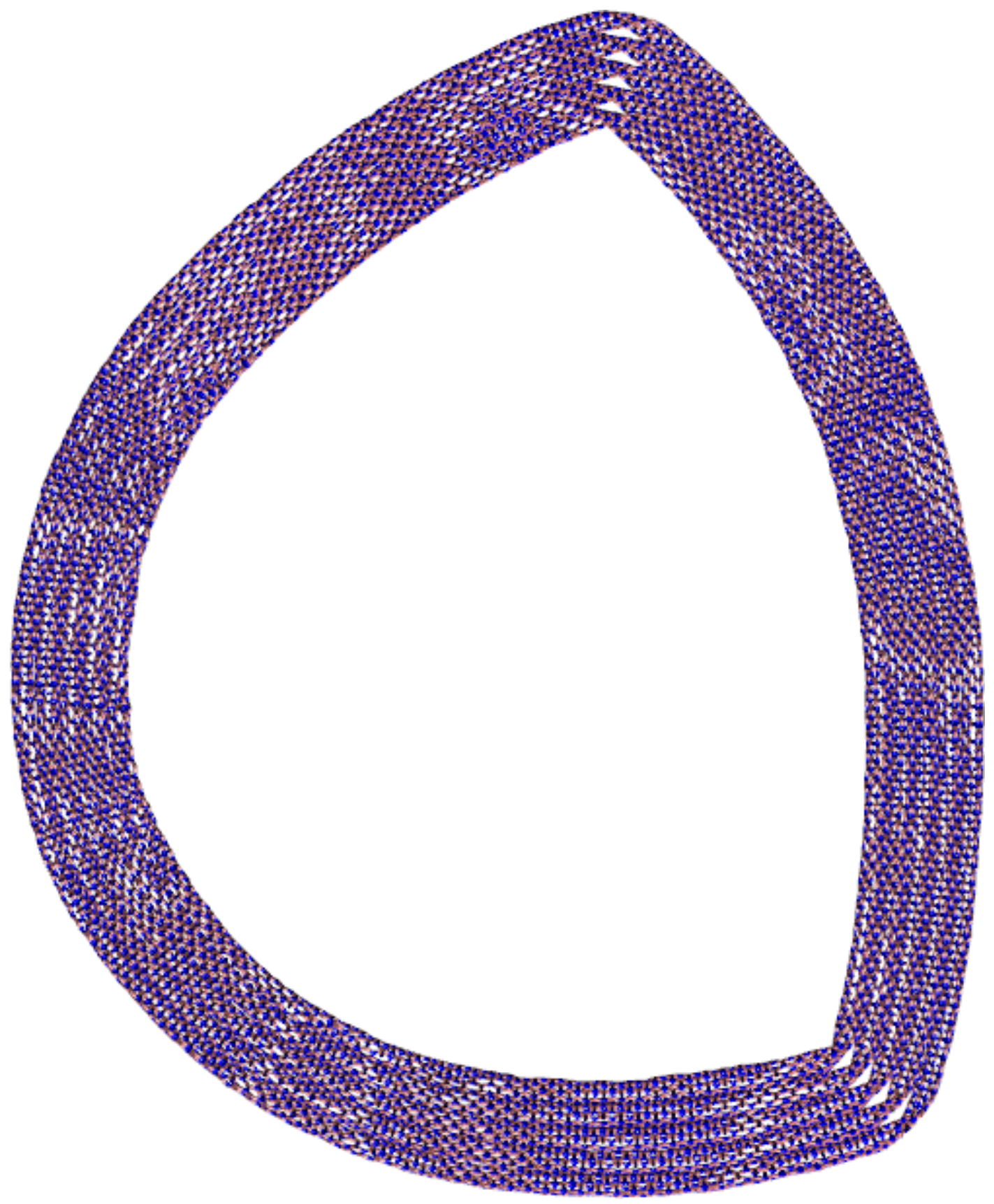




\section{References}

(1) Golberg, D.; Mitome, M.; Bando, Y.; Tang, C.; Zhi, C. Multi-walled boron nitride nanotubes composed of diverse cross-section and helix type shells. Appl. Phys. A 2007, $88,347-352$.

(2) Evans, D. J.; Holian, B. L. The Nose-Hoover thermostat. J. Chem. Phys. 1985, 83, 4069-4074.

(3) He, M.; Dong, J.; Zhang, K.; Ding, F.; Jiang, H.; Loiseau, A.; Lehtonen, J.; Kauppinen, E. I. Precise determination of the threshold diameter for a single-walled carbon nanotube to collapse. ACS Nano 2014, 8, 9657-9663.

(4) Impellizzeri, A.; Briddon, P.; Ewels, C. P. Stacking- and chirality-dependent collapse of single-walled carbon nanotubes: A large-scale density-functional study. Phys. Rev. B 2019, 100 . 